\title{
Designing a Residential Hybrid Electrical Energy Storage System Based on the Energy Buffering Strategy
}

\author{
Di Zhu†, Siyu Yue†, Yanzhi Wang†, Younghyun Kim‡, Naehyuck Changł, and Massoud Pedram† \\ $\dagger$ University of Southern California, CA, USA, $\$$ Seoul National University, Korea \\ $\dagger\{$ dizhu, siyuyue, yanzhiwa, pedram\}@usc.edu, $\ddagger\{y h k i m$, naehyuck\}@elpl.snu.ac.kr
}

\begin{abstract}
Due to severe variation in load demand over time, utility companies generally raise electrical energy price during periods of high load demand. A grid-connected hybrid electrical energy storage (HEES) system can help residential users lower their electric bills by storing energy during low-price hours and releasing the stored energy during high-price hours. A HEES system consists of different types of electrical energy storage (EES) elements, utilizing the benefits of each type while hiding their weaknesses. This paper presents a residential energy management system to maximize the annual profits on residential electric bills, based on a HEES system comprised of a lead-acid battery bank as the main storage bank and a Li-ion battery bank as the energy buffer. We first derive the optimal daily energy management policy based on energy buffering to minimize the daily energy cost. Next, we find the near-optimal design specifications of the energy management system, aiming at maximizing the amortized annual profits under practical constraints. We show that this system achieves averagely $11.10 \%$ more profits compared to the none-buffering HEES system.
\end{abstract}

\section{KEYWORDS}

Electric bill savings, energy management, hybrid electrical energy storage system.

\section{INTRODUCTION}

The electric energy demand generally ramps up significantly during certain hours of a day (a.k.a., peak hours), whereas the load demand following from the generation side is generally expensive and/or limited [1]. Hence, electric utility overprovisioning is often required in order to avoid blackouts during the peak hours. To incentivize users to perform some spontaneous energy management, i.e. demand side management (DSM), utility companies such as the Consolidated Edison Company of New York (conEdison) employ time-of-day pricing policy [2], with higher unit energy price during peak hours for residential users. Moreover, recent research on smart grid proposes real-time pricing policy [3], with electricity unit price as an increasing function of real-time energy demand.

Demand side management not only lowers the users' electric bills, but also reduces the demand on the peak power generation capability of utility companies. One way for the users to perform demand side management is to shift some of the residential load tasks from peak hours to off-peak hours [4][5]. This method has limited applicability since only a small fraction of the workloads is transferrable in time. A more practical method is to exploit electrical energy storage (EES) systems to store energy when the

This work is supported in part by the Software and Hardware Foundations program of the NSF's Directorate for Computer \& Information Science \& Engineering, the Center for Integrated Smart Sensors funded by the Ministry of Science, ICT \& Future Planning as Global Frontier Project (CISS- 2012054193). The SPORT lab at USC and ICT at SNU provides research facilities for this study. electricity price is low and supply energy for usage when the electricity price is high [6], and thereby, shift the households' energy demands from peak hours to off-peak hours.

In order to achieve high energy cost savings, an ideal EES system should possess many features such as high charge/discharge efficiency, high energy density, low cost per unit capacity and long cycle life [7]. Current EES system deployments are mainly homogeneous, i.e., they consist of a single type of EES element. A typical EES system may comprise of Lithium-ion (Liion) batteries, lead-acid batteries, supercapacitors or other types of EES elements. Nevertheless, none of the existing EES elements can simultaneously fulfill all the above desired features. Therefore the overall performance of a homogeneous EES system is limited by the underlying EES element characteristics, thereby restricting the application of EES systems for household uses.

Recently, a novel energy storage technology which has the potential to overcome these limitations, the hybrid EES (HEES) system, is gaining popularity [7][8]. A HEES system comprises of a number of heterogeneous EES elements and is therefore, if properly managed, able to exploit the strengths of each type of EES elements while hiding their weaknesses. The art of choosing an appropriate HEES management policy is a combined consideration of the charge/discharge efficiency of each EES element, capacity degradation, and expected service life of the HEES system. In addition, when the HEES system is applied to the household system, more practical factors must be considered, such as the capital cost and the energy density of EES banks, daily load profile, and the maintenance cost.

This paper presents a residential HEES system with an energy management policy based on the idea of energy buffering, exploiting the economic viability of the HEES system in household applications. After selecting the types of EES elements to be employed in the HEES system, we derive the optimal HEES management policy to minimize the daily energy cost, based on the idea of energy buffering. This policy is further improved by considering the longevity of the battery banks. With the results of the optimal HEES system daily control policy, we find the design specification of the HEES system, taking into consideration reallife factors such as the battery's capacity degradation, unit capital cost of EES elements, maintenance and replacing cost of the HEES system, etc. This HEES design optimization seeks to maximize the amortized annual profit under a monetary budget constraint and a volume constraint.

The rest of this paper is organized as follows. Related work and background are given in Sections 2 and 3, respectively. Section 4 describes the daily optimization of finding the optimal HEES charging/discharging management policy for daily energy cost reduction. Section 5 presents the global design optimization of maximizing the amortized annual profit by determining the HEES system specifications. The simulation results are shown in Section 6 and the paper is concluded in Section 7. 


\section{RELATED WORK}

There are two primary methodologies of demand side management, namely scheduling load demand and employing energy storage. As mentioned above, load demand scheduling has limited applicability because most of the user-specified tasks (e.g., watching TV and using air conditioner) can hardly be shifted to other time slots. Some other tasks also have timing limitations, e.g., washing machines may not be used during midnight due to noises.

Load shifting can also be achieved by utilizing electrical energy storage (EES) systems. For example, Exarchakos et al. study demand side management and profitability by optimizing the allocation of charge and discharge time of the EES system [9]. Wei et al. use a multi-agent system to model the demand side management problem with EES systems, and solve this problem using an adaptive learning approach [10]. These research efforts assume the EES systems have a fixed round-trip efficiency (cycle efficiency). However, the actual EES elements have variable round-trip efficiency due to the rate capacity effect, capacity degradation, self-discharge, etc.

The first research on the economic viability of a grid-connected HEES system is proposed by Zhu et al. [11]. Nevertheless, the proposed management policy overestimates the electric bill savings due to (i) the ignorance of capacity degradation of battery banks and (ii) the coarse-granularity of HEES management policy. To be more specific, first, the authors assume that leadacid battery bank and the Li-ion battery bank have constant capacity throughout their lifetime. Second, the management policy in [11] is hourly-based, which cannot reflect the fluctuation of the load profile. For example, a high peak load power consumption may last for only a couple of minutes, e.g. the usage of a microwave oven.

An important observation is that the peak hour load demand differs in different seasons. Figure 1 shows a daily demand profile from California Independent System Operator Corporation (ISO) [12]. Cooling during daytime in summer requires large energy consumption, thereby making the peak load power consumption in high season (i.e., summer) much higher than in low season (i.e., spring, fall, and winter). Therefore, we should develop different HEES management policies for different seasons.

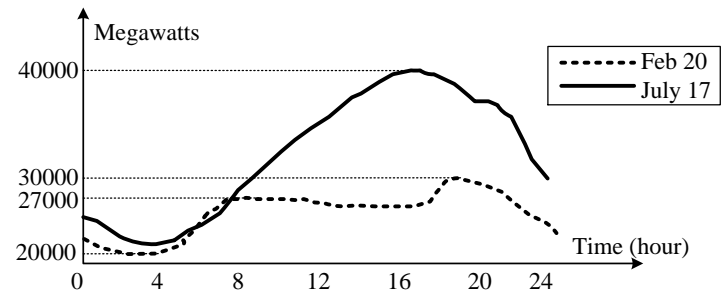

Figure 1. One-day power demand profiles in high season and low season.

\section{BACKGROUND}

We consider a scenario where a dwelling unit is equipped with an energy management system. This section first introduces two types of pricing policies, namely the time-of-day pricing and the real-time pricing. We then discuss some features of various EES elements that influence the economic viability of the HEES system. Finally, we choose the appropriate EES elements to build the proposed HEES system.

\subsection{Electricity Pricing Policies}

The unit energy price is defined as the price per unit energy consumption during a certain time slot. Because of the high electricity usage during peak hours, most utility companies provide customers with an alternative pricing policy, the time-ofday pricing, where the unit price varies in different periods of a day. In particular, the energy pricing policy in the New York City has the peak hour period from 10 a.m. to 10 p.m. and base hours for the others, high season from June to September and low season for the other months [2].

Recent research on Smart Gird proposes the real-time pricing policy, in which the unit energy price is a monotonically increasing function of the current load demand during peak hours [3]. During a time slot, the unit price increases with the increase of the total energy consumption, thereby discouraging high energy consumption. The proposed problem formulation is able to deal with all non-decreasing pricing functions. Without loss of generality, we assume two different real-time pricing policies in peak hours: (i) a discrete two-tier pricing function and (ii) a continuous increasing pricing function. During a certain time slot, the two-tier pricing asks for twice the regular unit price for the part of energy consumption that exceeds a certain threshold during a time slot, and the continuous pricing asks for a unit price proportional to the energy consumption to the power of 0.4 , i.e. unit energy price $u(E)=u_{0} E^{0.4}$, where $E$ is the energy consumption during the time slot, and $u_{0}$ is a constant. We define $c(E)=u(E) \cdot E$ as the total cost of the energy consumption $E$ during this time slot. The peak hour cost functions of the two realtime pricing policies as well as the time-of-day pricing policy in the New York City are shown in Figure 2.

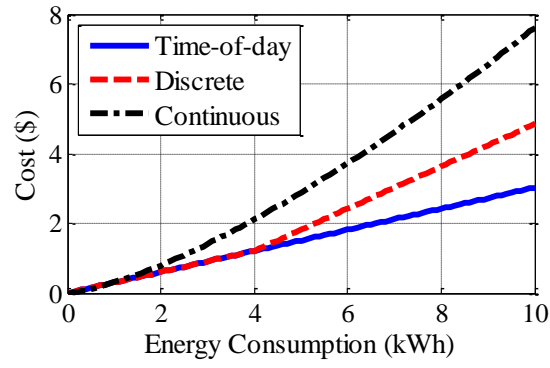

Figure 2. Peak hour cost functions of various pricing policies.

\subsection{Performance Metrics of EES Elements}

Common commercial off-the-shelf EES elements include leadacid batteries, Lithium-ion (Li-ion) batteries, NiMH batteries, metal-air batteries and supercapacitors. Table 1 compares five performance metrics of these batteries, which are essential to our residential energy management system. We highlight the strengths of each type of EES elements in boldface and the weaknesses in italic. They are further elaborated in the following.

\subsubsection{Capital Cost}

Before making a purchase, consumers must be convinced that the energy management system is able to bring more savings on their electric bills than the system's cost. Capital cost of the EES elements employed in the system directly affects its profitability.

For batteries, we define the unit price of a battery to be the dollar cost of the battery divided by its nominal full-charge capacity $Q$ (in $\mathrm{Ah}$ ) and terminal voltage $V$ :

$$
p=\frac{\text { Dollar Cost }}{Q V}(\$ / \mathrm{Wh})
$$


Table 1. Performance Metrics of Various EES Elements.

\begin{tabular}{|c|c|c|c|c|c|}
\hline & Capital cost $(\$ / \mathrm{kWh})$ & Cycle efficiency & Cycle life & Self-discharge per day & Energy density (Wh/L) \\
\hline Lead-acid batteries & $100-200$ & $70-90 \%$ & $500-800$ & $0.1-0.3 \%$ & $60-75$ \\
\hline NiMH batteries & $450-1,000$ & $66 \%$ & $500-1,000$ & $0.5-1 \%$ & $150-350$ \\
\hline Li-ion batteries & $600-2,500$ & $>90 \%$ & 1,000-10,000+ & $0.1-0.3 \%$ & $250-700$ \\
\hline Metal-Air batteries & $10-60$ & $<50 \%$ & $100-300$ & Very small & 1.5k-10k \\
\hline Supercapacitor & $20,000-50,000$ & $>90 \%$ & $50,000+$ & $20-40 \%$ & Around 10 \\
\hline
\end{tabular}

Currently, lead-acid batteries and metal-air batteries are among the cheapest types of EES elements, while Li-ion batteries and $\mathrm{NiMH}$ batteries have four times the unit price or even higher. Compared with these batteries, supercapacitors have a significantly high unit price as shown in Table 1 .

\subsubsection{Self-Discharge Effect}

Supercapacitors suffer from severe self-discharge effect: They continuously lose stored energy regardless of whether they are connected to a load or not. Similar to a normal capacitor, the amount of energy stored in a supercapacitor is proportional to the square of its open circuit terminal voltage (i.e., $E=C V^{2} / 2$ ). When no load is connected to it, a supercapacitor may lose $20 \% \sim 40 \%$ of its stored energy in one day [7]. The supercapacitor voltage decay after $\Delta t$ time is given by:

$$
V^{s c}(t+\Delta t)=V^{s c}(t) e^{-\frac{\Delta t}{\tau}}
$$

where $\tau$ is the self-discharge time constant.

\subsubsection{Cycle Efficiency}

The actual performance of the energy storage system is greatly dependent on the discharge efficiency of EES elements. The discharge efficiency of an EES element is defined as the ratio of the battery's output current to the actual degradation rate of its stored charge. One of the major factors that affects the discharge efficiency is the rate capacity effect. It specifies the fact that the actual rate of charge loss $I_{e q}$ inside a battery is a superlinear function of its output discharge current $I_{\text {disch }}$ [13]:

$$
I_{e q}=\left(\frac{I_{\text {disch }}}{I_{\text {ref }}}\right)^{k} I_{\text {ref }}
$$

where $I_{\text {ref }}$ is the reference discharge current, and if not explicitly provided, it is the constant current that takes 20 hours to fully discharge the battery with nominal full-charge capacity $Q$ (in Ah), i.e., $I_{\text {ref }}=Q / 20$. The constant $k$ affects the actual efficiency of the discharging process. For lead-acid batteries, $k(1.3$ to 1.4$)$ is much higher than that of Li-ion batteries (less than 1.1) according to the battery characterization results in our lab. Supercapacitors have negligible rate capacity effect, i.e. $k=1$.

\subsubsection{Energy Density}

The energy density is the amount of stored energy per unit volume or weight. As shown in Table 1, Li-ion batteries and Metal-air batteries have higher energy density compared to leadacid batteries, while supercapacitors have generally lower density than electrochemical batteries.

\subsubsection{Cycle Life}

The cycle life is another important performance metric of EES elements, defined as the number of cycles an EES element can perform before its capacity drops to a specific percentage $(80 \%$ typically) of its initial full-charge capacity. The cycle life directly affects the HEES system service lifetime. Metal-air, NiMH and lead-acid batteries have significantly lower lifetime compared to Li-ion batteries and supercapacitors.

\subsection{HEES System}

Based on the above performance metrics, we choose lead-acid batteries as the main energy storage bank and Li-ion batteries as the energy buffer. The main reasons are:

1) The main storage bank requires both low cost for large capacity and acceptable cycle efficiency. Lead-acid batteries are one of the most developed types of EES elements with low capital cost. Although metal-air batteries have even lower capital cost, it has significantly lower cycle efficiency (less than 50\%) and shorter lifetime.

2) An energy buffer is deployed in the energy management system to compensate for the rate capacity effect of leadacid batteries. Supercapacitors and $\mathrm{Li}$-ion batteries both have high cycle efficiency and long lifetime, and are therefore able to be adopted as energy buffer. The former, however, has very high capital cost (an average of $\$ 40,000 / \mathrm{kWh}$ ), impractical for the HEES system to make profits under current technology. Therefore, we deploy Liion batteries to perform energy buffering.

Figure 3 shows the structure of a typical grid-connected HEES system. Without loss of generality, we assume one lead-acid battery bank and one Li-ion battery bank in the HEES system. The actual deployment of a battery bank may include multiple cells in parallel. We take the Li-ion battery bank as an example. Suppose there are $m \mathrm{Li}$-ion battery cells in parallel, each with the capacity of $\frac{Q_{x}}{m}$ and discharge current $\frac{I_{d i s}}{m}$, forming a bank with an overall capacity of $Q_{x}$ and an overall discharge current $I_{\text {dis }}$. The reference current of a single battery cell is $\frac{Q_{x}}{20 m}$. Hence, the actual charge loss rate of a single cell is

$$
I_{e q, \operatorname{sing}}=\left(\frac{I_{d i s}}{m} \cdot \frac{20 m}{Q_{x}}\right)^{k} \frac{Q_{x}}{20 m}=\left(\frac{20 I_{d i s}}{Q_{x}}\right)^{k} \frac{Q_{x}}{20 m}
$$

The overall charge loss rate of the bank is $I_{e q, \operatorname{sing}} \cdot m=$ $\left(\frac{20 I_{d i s}}{Q_{x}}\right)^{k} \frac{Q_{x}}{20}$. Therefore, these $m$ cells in parallel each with the capacity of $\frac{Q_{x}}{m}$ are equivalent to one big cell with the capacity of $Q_{x}$ in terms of calculating the discharge efficiency.

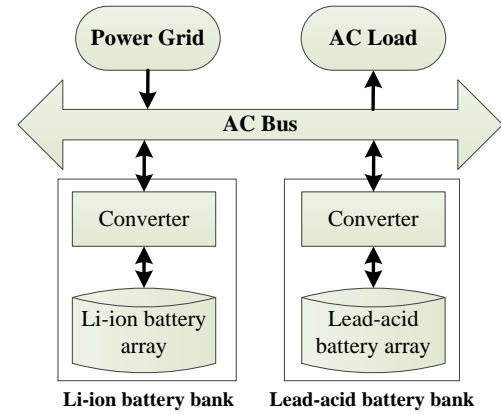

Figure 3. HEES system structure.

In addition, we need to employ various power conversion circuits in our system structure since we use batteries to supply 
power for AC loads. These power conversion circuits are nonideal and introduce certain amount of power dissipation due to their internal resistance and switching power losses.

\section{DAILY ENERGY MANAGEMENT}

This section formulates and solves the problem of developing an energy management policy to achieve maximum daily energy cost reduction under a given HEES system specification (i.e., EES capacities) and load electricity consumption profile. We refer to this problem as the "daily-cost-reduction problem" or the DCR problem for short. Next we adjust the optimization result by adding limits to the usable capacity of battery banks in order to prolong the lifetime of EES banks.

\subsection{Energy Buffering Strategy}

The daily energy management policy controls the charge/discharge currents of each EES banks, aiming at maximizing the daily energy cost saving. To achieve this goal, one intuitive thought is to fully charge the batteries during base hours and release all the stored energy during peak hours. However, this is not always the optimal for the following three reasons.

First, for the lead-acid batteries which suffer from severe rate capacity effect, according to (3), the actual charge loss rate inside a battery is a superlinear function of its discharge current. The cycle efficiency decreases with the increase of discharge current. The rate capacity effect might grow so severe that increasing discharge current merely adds up to the energy cost.

Second, selling electrical energy back to the grid is not allowed in our problem formulation, since most companies do not repurchase the stored electricity in EES systems. In other words, the delivered power of the battery banks cannot be larger than the load demand.

Third, we know from Figure 1 that the load power consumption during peak hours may differ significantly in different days. The amount of energy stored in the lead-acid battery bank during base hours should thus depend on the peak hour energy demand instead of always using the full capacity.

We show that the fluctuation of load demand greatly limits the discharge efficiency of the HEES system. Let us consider 50 leadacid batteries in parallel, each with an energy capacity of $1 \mathrm{kWh}$. Figure 4 shows a 4-hour load power profile during peak hours as well as the curves of the lead-acid battery bank's delivered power. Previous research [11] does not allow the Li-ion battery bank to be charged during peak hours. In other words, the delivered power of the lead-acid battery bank must be lower than the load demand (the no-buff curve). The actual energy loss in the lead-acid battery bank is $3.833 \mathrm{kWh}$ in this four-hour duration, $8.67 \%$ more than the energy loss of $3.528 \mathrm{kWh}$ when ideal (constant) discharge current is applied to the battery bank to deliver the same amount of energy.

This leads to the energy management policy which uses the Liion battery bank as a "buffer": The Li-ion battery bank can be charged by the lead-acid battery bank when the load demand is low. In this way, the ideal lead-acid discharge current can be achieved by employing the energy buffering scheme: at demand valleys (e.g., during $2 \mathrm{~h}$ to $2.7 \mathrm{~h}$ in Figure 4 ), the lead-acid battery bank provides energy both to the load devices and the Li-ion battery bank. The energy stored in the Li-ion battery bank is released when load demand peaks arrive (e.g., during $3 \mathrm{~h}$ to $3.3 \mathrm{~h}$ in Figure 4).

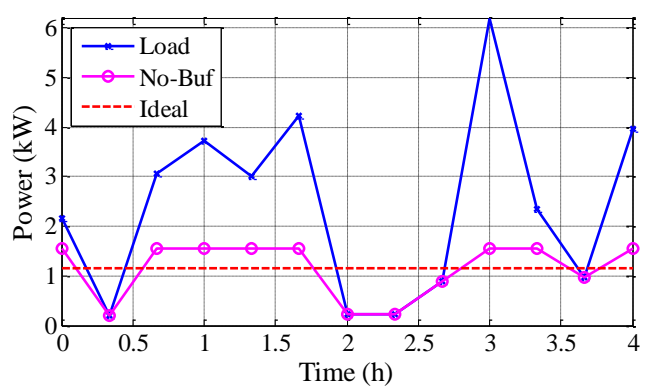

Figure 4. Delivered power of the lead-acid battery bank with and without energy buffering.

\subsection{DCR Problem Formulation and Solution}

We use $x$ in the parameters of Li-ion batteries and $y$ for leadacid batteries hereinafter. The DCR problem aims at deriving the daily HEES management policy to minimize the daily energy cost with the given two battery banks: Li-ion battery bank with capacity $Q_{x}$ and terminal voltage $V_{x}$, and lead-acid battery bank with capacity $Q_{y}$ and terminal voltage $V_{y}$.

Instead of an hourly-based strategy, we use $N$ decisions epochs (and hence $N$ time slots) per hour in the proposed HEES management algorithm. The index set of the decision epochs in the peak hours is denoted by $P K=\left\{t_{1} N+1, t_{1} N+2, \ldots, t_{2} N\right\}$, and the index set in the base hours is denoted by $B S=$ $\left\{1,2, \ldots, t_{1} N\right\} \cup\left\{t_{2} N+1, t_{2} N+2, \ldots, 24 N\right\}$, where $t_{1}$ and $t_{2}$ are the start and end time (in hours) of peak hours.

Let $x_{t_{1} N+1}, \ldots, x_{t_{2} N}$ denote the Li-ion battery bank charge/discharge current in the corresponding time slots of peak hours and $y_{t_{1} N+1}, \ldots, y_{t_{2} N}$ denote the lead-acid battery bank charge/discharge current. These value of the currents are the optimization variables. They are positive when the battery banks are being discharged, and negative otherwise. The objective is to maximize the daily energy cost saving, which is defined by the original residential energy cost without the HEES system subtracted by the new energy cost with the HEES system. This energy cost saving is comprised of two parts:

1) The energy cost reduction during the peak hours:

where

$$
\sum_{i \in P K}\left(c_{P K}\left(E_{i}^{\text {load }}\right)-c_{P K}\left(E_{i}^{\text {load }}-\Delta E_{i}^{\text {load }}\right)\right)
$$

$$
\Delta E_{i}^{\text {load }}=\left\{\begin{array}{c}
\left(\eta_{1} x_{i} V_{x}+\eta_{1} y_{i} V_{y}\right) / N, \text { if } x_{i} \geq 0 \\
\left(x_{i} V_{x} / \eta_{2}+\eta_{1} y_{i} V_{y}\right) / N, \text { if } x_{i}<0
\end{array}\right.
$$

where $c_{P K}(E)$ is the cost of the energy consumption $E$ in a time slot during the peak hours. $E_{i}^{\text {load }}$ is the load energy demand, $\Delta E_{i}^{\text {load }}$ is the energy provided by the battery banks, and $\eta_{1}$ and $\eta_{2}$ denote the power conversion efficiency of the DC-AC inverter and the AC-DC rectifier, respectively. $y_{i}$ is non-negative during the peak hours, whereas $x_{i}$ can be negative because the Li-ion battery bank may be charged during peak hours.

2) The additional energy cost from charging the battery banks during base hours, given by

$$
-\sum_{i \in B S} c_{B S}\left(\frac{x_{c} V_{x}+y_{c} V_{y}}{\eta_{2}}\right)
$$

where $c_{B S}(E)=u_{B S} \cdot E$ is the base hour energy cost function in a time slot and $u_{B S}$ is the base hour unit energy price. Here $x_{C}$ and $y_{c}$ are the constant charging currents of the Li-ion battery bank and the lead-acid battery bank during base hours, respectively. They are calculated by

$$
x_{c}=\frac{1}{24-t_{2}+t_{1}} \sum_{i \in P K} \Delta Q_{i}
$$




$$
y_{c}=\frac{1}{24-t_{2}+t_{1}} \sum_{i \in P K}\left(\frac{y_{i}}{I_{y, r e f}}\right)^{k_{y}} I_{y, r e f} / N
$$

where $24-t_{2}+t_{1}$ refers to the duration of base hours. $\left(\frac{y_{i}}{I_{y, \text { ref }}}\right)^{k_{y}} I_{y, \text { ref }} / N$ is the charge loss of the lead-acid battery bank during the $i$-th time slot in peak hours, calculated by

$$
\left(\frac{y_{i}}{I_{y, \text { ref }}}\right)^{k_{y}} I_{y, \text { ref }} / N=\left(\frac{20 y_{i}}{Q_{y}}\right)^{k_{y}} \frac{Q_{y}}{20} / N, \forall i \in P K
$$

In (8), during the $i$-th time slot in peak hours, $\Delta Q_{i}$ is the amount of loss/accumulation of charge stored in the Li-ion battery bank. $\Delta Q_{i}$ is positive if the battery bank is being discharged during the $i$-th time slot, and negative otherwise. Hence, $\sum_{i \in P K} \Delta Q_{i}$ is the initial charge stored in the Li-ion battery bank at the beginning of the peak hours. When the bank is being charged, i.e., $x_{i}<0, \Delta Q_{i}$ is calculated by the current multiplied by duration: $\Delta Q_{i}=x_{i} / N$. When the bank is being discharged, i.e., $x_{i} \geq 0$, the $\Delta Q_{i}$ value is calculated by

$$
\Delta Q_{i}=\left(\frac{x_{i}}{I_{x, \text { ref }}}\right)^{k_{x}} I_{x, \text { ref }} / N=\left(\frac{20 x_{i}}{Q_{x}}\right)^{k_{x}} \frac{Q_{x}}{20} / N
$$

The HEES management policy must satisfy three constraints: the load demand constraint, Li-ion battery capacity constraint, and lead-acid battery capacity constraint. The load demand constraint implies that the energy provided by the HEES system at any time slot cannot exceed the load demand, i.e., $E_{i}^{\text {load }} \geq \Delta E_{i}^{\text {load }}, \forall i \in$ $P K$.

The Li-ion battery capacity constraint says that the amount of charge stored in the Li-ion battery bank should be, during the whole operation time, within the range of 0 and $Q_{x}$. The remaining charge at the end of the $l$-th time slot is expressed by

$$
0 \leq \sum_{i \in P K} \Delta Q_{i}-\sum_{i=t_{1} N+1}^{l} \Delta Q_{i} \leq Q_{x}, \forall l \in P K
$$

where $\sum_{i \in P K} \Delta Q_{i}$ is the initial charge stored in the Li-ion battery bank at the beginning of peak hours, and $\sum_{i=t_{1} N+1}^{l} \Delta Q_{i}$ stands for the total charge change from the beginning of the peak hours to the end of the $l$-th time slot.

For the lead-acid battery bank, the discharge currents should also satisfy the capacity constraint. The lead-acid battery bank can only be discharged $\left(y_{i} \geq 0\right)$ during peak hours, and the total charge loss should be no more than the capacity $Q_{y}$.

The DCR problem is therefore formulated as follows:

\section{Given:}

1) Battery bank capacities (in Ah) $Q_{x}, Q_{y}$;

2) Battery terminal voltages $V_{x}, V_{y}$;

3) The peak hour energy cost function $c_{P K}(E)$;

4) The base hour unit energy price $u_{B S}$;

5) Residential load energy profile $E_{i}^{\text {load }}, i=1, \ldots, 24 N$;

6) Batteries' rate capacity effect coefficients $k_{x}, k_{y}$;

7) DC-AC inverters' power conversion efficiency $\eta_{1}$ and ACDC rectifier's efficiency $\eta_{2}$.

Find: Discharge current profiles $x_{t_{1} N+1}, \ldots, x_{t_{2} N}, y_{t_{1} N+1}, \ldots, y_{t_{2} N}$ of the two battery banks during peak hours.

Maximize: The daily energy cost reduction:

$$
\begin{gathered}
\operatorname{DCR}\left(x_{t_{1} N+1}, \ldots, x_{t_{2} N}, y_{t_{1} N+1}, \ldots, y_{t_{2} N}\right) \\
=\sum_{i \in P K}\left(c_{P K}\left(E_{i}^{\text {load }}\right)-c_{P K}\left(E_{i}^{\text {load }}-\Delta E_{i}^{\text {load }}\right)\right) \\
-\sum_{i \in B S} u_{B S} \cdot \frac{x_{c} V_{x}+y_{c} V_{y}}{\eta_{2}} \\
\text { where } \Delta E_{i}^{\text {load }}, x_{c} \text {, and } y_{c} \text { are given by (6), (8), and (9). }
\end{gathered}
$$

\section{Subject to:}

1) The load energy constraint:

$$
E_{i}^{\text {load }}-\Delta E_{i}^{\text {load }} \geq 0, \forall i \in P K
$$

2) The Li-ion battery capacity constraint:

$$
\begin{gathered}
0 \leq \sum_{i \in P K} \Delta Q_{i} \leq Q_{x} \\
0 \leq \sum_{i \in P K} \Delta Q_{i}-\sum_{i=t_{1} N+1}^{l} \Delta Q_{i} \leq Q_{x}, \forall l \in P K
\end{gathered}
$$

where $\Delta Q_{i}=\left\{\begin{array}{ll}\left(\frac{20 x_{i}}{Q_{x}}\right)^{k} \frac{Q_{x}}{20} / N, & \text { if } x_{i} \geq 0 \\ x_{i} / N, & \text { if } x_{i}<0\end{array}\right.$.

3) The lead-acid battery capacity constraint:

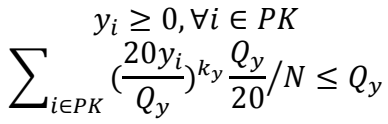

The above DCR problem is non-convex optimization. To effectively solve this problem, we first adopt a heuristic method to determine when the Li-ion battery bank is being charged (i.e. to determine the signs of the $x_{i}$ values). More precisely, we first assume that the lead-acid battery bank is discharged using current $y_{d i s}$. This value is introduced merely to determine the signs of $x_{i}$. Then we add the constraints of the charge/discharge status of the Li-ion battery bank during the $i$-th time slot, determined as:

$$
\left\{\begin{array}{rr}
x_{i} \geq 0, & \text { if } \eta_{1} y_{d i s} V_{y} / N \leq E_{i}^{\text {load }}, \\
x_{i} \leq 0, & \text { otherwise. }
\end{array},\right.
$$

Once the signs of $x_{i}$ are determined, the solution is calculated by the MatLab optimization tool fmincon [14]. The initial value of $y_{\text {dis }}$ is obtained by solving the equation: $Q_{y}=\left(t_{2}-\right.$ $\left.t_{1}\right)\left(\frac{20 y_{d i s}}{Q_{y}}\right)^{k_{y}} \frac{Q_{y}}{20}$. We adjust $y_{\text {dis }}$ incrementally and record the best solution with the maximum energy cost saving during the day.

The maximum energy cost reduction of the $j$-th day is expressed as a function $f_{j}\left(Q_{x}, Q_{y}\right)$ of the Li-ion battery bank capacity $Q_{x}$ and the lead-acid battery bank capacity $Q_{y}$. By summing up the daily results, we get the maximum seasonal cost reduction function of low season and high season:

$$
\begin{aligned}
& F_{L S}\left(Q_{x}, Q_{y}\right)=\sum_{j \in L S} f_{j}\left(Q_{x}, Q_{y}\right) \\
& F_{H S}\left(Q_{x}, Q_{y}\right)=\sum_{j \in H S} f_{j}\left(Q_{x}, Q_{y}\right)
\end{aligned}
$$

The results are stored in a two-dimensional look-up table (LUT). This LUT is used to determine the specification of the residential HEES system, i.e., before actually acquiring the system. In other words, this is a one-time calculation for residential users. After acquiring the system, the residential users merely need to solve the DCR problem once a day, of which the runtime is in the scale of several seconds.

\subsection{Lifetime-Aware DCR Problem and Solution}

The optimal solution of the above DCR problem assumes that the HEES system is allowed to fully charge and discharge both battery banks during off-peak hours and peak hours, respectively. Nevertheless, fully charging and discharging of batteries result in a fast capacity degradation rate, and thereby, significantly shorten the battery lifetime [15][18]. Allowing only a portion of the total capacities to be used for charging and discharging seems to "waste" part of the battery bank capacity, but may gain more benefits from the extended battery bank lifetime. Therefore, we should reconsider the DCR problem based on the lifetime characterization of Li-ion batteries and lead-acid batteries. 
For Li-ion batteries, we adopt the lifetime model proposed in [15]. The state-of-charge (SoC) of a battery is defined as the ratio of the remaining charge to its full charge capacity (FCC). According to this model, the lifetime of a Li-ion battery can be superlinearly extended by lowering the average SoC and/or minimizing the SoC swing in the charge/discharge cycles. Let $s_{x}$ (in percentage) denote the limit on the maximum SoC swing of the Li-ion battery bank. With this constraint, the HEES management policy should limit the Li-ion battery bank to operate within the range of a minimum $\mathrm{SoC}$ of 0 and the maximum SoC of $s_{x}$, since this policy also minimizes the average SoC level. For example, if we limit the usable capacity to be $70 \%$ of the total capacity, the control policy should not charge the Li-ion battery bank to more than $70 \%$ of its full capacity, which is equivalent to an SoC swing limit of $70 \%$ and an average SoC level of $35 \%$ assuming constant charge and discharge. Figure 5(a) shows the Li-ion battery lifetime in charge/discharge cycles as a function of the maximum SoC swing.

For lead-acid batteries, we use the Ah-Throughput model described in [18]. The Ah-Throughput model assumes that a fixed amount of energy can be cycled through a lead-acid battery during its lifetime. Other sources of data ${ }^{1}$ also match the inversely proportional relationship between depth of discharge (DoD) of lead-acid batteries and cycle numbers. The DoD is defined differently from SoC swing. If the maximum DoD of a lead-acid battery is set to be 0.7 , it cannot be discharged to less than $30 \%$ SoC. Figure 5(b) shows the lead-acid battery lifetime in cycles as a function of the maximum DoD.
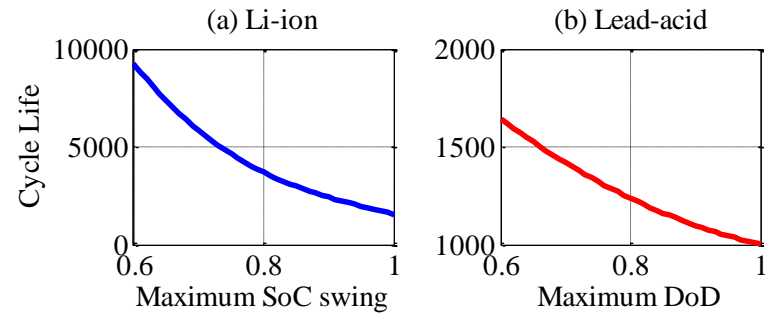

Figure 5. Battery lifetime as a function of maximum SoC swing/DoD.

Let us consider the maximum SoC swing of the Li-ion battery bank is $s_{x}$ and the maximum DoD of the lead-acid battery bank is $s_{y}$. The Li-ion battery bank capacity constraint in the original DCR problem formulation should be changed accordingly:

$$
\begin{gathered}
0 \leq \sum_{i \in P K} \Delta Q_{i} \leq s_{x} Q_{x} \\
0 \leq \sum_{i \in P K} \Delta Q_{i}-\sum_{i=t_{1} N+1}^{l} \Delta Q_{i} \leq s_{x} Q_{x}, \forall l \in P K
\end{gathered}
$$

where $\Delta Q_{i}$ is the same with that in (11).

The lead-acid battery bank constraint should be changed into:

$$
\sum_{i \in P K}\left(\frac{20 y_{i}}{Q_{y}}\right)^{k_{y}} \frac{Q_{y}}{20} / N \leq s_{y} Q_{y} .
$$

Next, we discuss how to solve the lifetime-aware DCR problem. How to choose a proper maximum SoC swing and DoD to maximize the overall profit is discussed in Section 5.

Let $\hat{f}_{j}\left(Q_{x}, Q_{y}, s_{x}, s_{y}\right)$ denote the maximum daily energy cost reduction of the $j$-th day achieved by solving the lifetime-aware DCR problem. Storing the results of the lifetime-aware DCR problem require a four-dimensional LUT which is too costly. In the following, we reduce the storage space to a two-dimensional

\footnotetext{
${ }^{1}$ http://pvedrom.pveducation.org/BATTERY/charlead.htm
}

LUT by finding a solution of the lifetime-aware DCR problem using the original DCR problem. We use $f_{j}\left(s_{x} Q_{x}, s_{y}{ }^{1 / k_{y}} Q_{y}\right)$ as an approximation of the maximum daily energy cost reduction $\hat{f}_{j}\left(Q_{x}, Q_{y}, s_{x}, s_{y}\right)$, and prove that $f_{j}\left(s_{x} Q_{x}, s_{y}{ }^{1 / k_{y}} Q_{y}\right)$ is an underestimation of $\hat{f}_{j}\left(Q_{x}, Q_{y}, s_{x}, s_{y}\right)$, i.e.,

$$
\hat{f}_{j}\left(Q_{x}, Q_{y}, s_{x}, s_{y}\right) \geq f_{j}\left(s_{x} Q_{x}, s_{y}{ }^{1 / k_{y}} Q_{y}\right) \text {. }
$$

Proof: Let $x_{t_{1} N+1}, \ldots, x_{t_{2} N}, y_{t_{1} N+1}, \ldots, y_{t_{2} N}$ and $q_{x}^{\text {ini }}$ be the optimal solution of the original DCR problem with Li-ion and Lead-acid capacity equal to $s_{x} Q_{x}$ and $s_{y}{ }^{1 / k_{y}} Q_{y}$, respectively. Let,

$$
g\left(x_{i}\right)= \begin{cases}x_{i}, & x_{i} \geq 0 \\ x_{i} s_{x}{ }^{k}{ }^{-1}, & x_{i}<0\end{cases}
$$

If we replace $x_{i}$ with $g\left(x_{i}\right)$ in the objective function $D C R$, the same amount of energy is provided to the load while less charge is taken from the grid, which can be expressed by

$\operatorname{DCR}\left(g\left(x_{t_{1} N+1}\right), \ldots, g\left(x_{t_{2} N}\right), y_{t_{1} N+1}, \ldots, y_{t_{2} N}\right)$

$\geq \operatorname{DCR}\left(x_{t_{1} N+1}, \ldots, x_{t_{2} N}, y_{t_{1} N+1}, \ldots, y_{t_{2} N}\right)$.

Furthermore, $g\left(x_{t_{1} N+1}\right), \ldots, g\left(x_{t_{2} N}\right), y_{t_{1} N+1}, \ldots, y_{t_{2} N}$ satisfy the constraints of the lifetime-aware DCR problem with variables $\left(Q_{x}, Q_{y}, S_{x}, S_{y}\right)$. This means that they are a set of feasible solutions of the lifetime-aware DCR problem, of which the DCR result must be smaller than or equal to the result of optimal solution $\hat{f}_{i}\left(Q_{x}, Q_{y}, s_{x}, s_{y}\right)$, i.e.,

$\hat{f}_{i}\left(Q_{x}, Q_{y}, s_{x}, s_{y}\right) \geq \operatorname{DCR}\left(g\left(x_{t_{1} N+1}\right), \ldots, g\left(x_{t_{2} N}\right), y_{t_{1} N+1}, \ldots, y_{t_{2} N}\right)$

Combining (27) and (28), we have

$$
\hat{f}_{i}\left(Q_{x}, Q_{y}, s_{x}, s_{y}\right)=f_{i}\left(s_{x} Q_{x}, s_{y}{ }^{1 / k_{y}} Q_{y}\right)
$$

This proof indicates that we underestimate the actual energy cost reduction by using the solution of original DCR problem to approximate the lifetime-aware DCR problem. We randomly pick different days for the experiments, and the simulation results show that the estimation error is less than 3.4\%. Table 2 shows the simulation results of four days with different EES bank capacities, SoC swing and $\mathrm{DoD}$ values.

Table 2. Percentage error in approximation in different days

\begin{tabular}{|c|c|c|c|c|c|c|c|}
\hline Day & $\boldsymbol{Q}_{\boldsymbol{x}}$ & $\boldsymbol{Q}_{\boldsymbol{y}}$ & $\boldsymbol{s}_{\boldsymbol{x}}$ & $\boldsymbol{s}_{\boldsymbol{y}}$ & $\boldsymbol{f}_{\boldsymbol{i}}$ & $\hat{\boldsymbol{f}}_{\boldsymbol{i}}$ & error \\
\hline 1 & 500 & 3000 & 0.6 & 0.6 & 2.1198 & 2.1906 & $3.34 \%$ \\
\hline 160 & 500 & 2000 & 0.5 & 0.5 & 4.2199 & 4.2960 & $1.80 \%$ \\
\hline 201 & 1000 & 2000 & 0.8 & 0.7 & 6.7788 & 6.8500 & $1.05 \%$ \\
\hline 360 & 100 & 500 & 0.5 & 0.5 & 0.3373 & 0.3469 & $2.85 \%$ \\
\hline
\end{tabular}

Similarly, the approximated values of the lifetime-aware maximum seasonal energy cost reductions satisfy:

$$
\begin{aligned}
& \widehat{F_{L}}\left(Q_{x}, Q_{y}, s_{x L}, s_{y L}\right) \geq F_{L}\left(s_{x L} Q_{x}, s_{y L}{ }^{1 / k_{y}} Q_{y}\right), \\
& \widehat{F_{H}}\left(Q_{x}, Q_{y}, s_{x H}, s_{y H}\right) \geq F_{H}\left(s_{x H} Q_{x}, s_{y H}{ }^{1 / k_{y}} Q_{y}\right) .
\end{aligned}
$$

where $s_{x L}, s_{y L}$ is the maximum SoC swing of the Li-ion battery bank and the maximum DoD of the lead-acid battery bank in low season, respectively. Similarly, $s_{x H}$ and $s_{y H}$ are the corresponding variables for high season.

\section{ANNUAL PROFIT MAXIMIZATION}

This section discusses the problem of finding the optimal design specification of the HEES system, i.e., the capacities and maximum SoC swings of both battery banks, which maximizes the amortized annual profit on electric bills.

\subsection{Capacity Degradation}

Capacity degradation describes the fact that the effective FCC of a battery gradually drops cycle by cycle. When its capacity drops to a specific percentage ( $80 \%$ typically) of its initial FCC, 
the battery is assumed to reach its end-of-life [7]. For lead-acid batteries, we assume constant degradation rate:

$$
\Delta L_{y}=\frac{0.2 \times E_{\text {cycled }}}{E_{\text {total }}}
$$

where $E_{\text {cycled }}$ is the energy the lead-acid battery has cycled and $E_{\text {total }}$ is the total energy it can cycle throughout its lifetime.

The Li-ion battery degradation is a function of the number of finished charge/discharge cycles and SoC swing as described in Section 4.3. According to the DCR problem, the lead-acid battery bank experiences one charge/discharge cycle per day. But for the Li-ion battery bank, the number of cycles in a day depends on the optimization result of the DCR problem. We estimate the number $\omega$ of Li-ion battery bank charge/discharge cycles in one day by the following equation:

$$
\omega\left(Q_{x}, Q_{y}, s_{x}, s_{y}\right)=\frac{1}{Q_{x}} \sum_{i \in P K}\left|x_{i}\right| / N+1
$$

The left-hand side denotes the cycled energy within this day, including both peak hours and base hours. On the right-hand side, we add up the absolute values of Li-ion battery bank charge/discharge flow, assuming that the initial charge of the Liion battery bank is $Q_{x}$ (fully charged) at the beginning of peak hours. The power conversion efficiency and the rate capacity effect are not considered, since (i) the errors caused by the former approximately cancel out each other during charge and discharge, and (ii) the rate capacity effect of the Li-ion batteries is insignificant compared to the lead-acid batteries. With the daily cycle number $\omega$ for each day, we calculate the Li-ion battery capacity degradation $\Delta L_{s x}$ using the model described in [15].

Since our optimization framework allows for low usage of Liion batteries (the maximum SoC swing can be lower than 0.6), we must take the calendar life of the Li-ion battery bank into consideration, which describes the battery bank capacity degradation as a result of the passage of time [16]. We adopt the Li-ion battery calendar life prediction model in [17]:

$$
d=e^{(4661 / T-14)} \Delta L_{c x}{ }^{2}+e^{(4437 / T-11.6)} \Delta L_{c x}
$$

where $d$ is time in days and $T$ is temperature in Kelvin. Li-ion batteries experience the minimum capacity degradation of $\Delta L_{c x}$ after $d$ days no matter how much it is used. Combined with the $\Delta L_{s x}$ value predicted by [15], we have:

$$
\Delta L_{x}=\max \left(\Delta L_{c x}, \Delta L_{s x}\right)
$$

\subsection{Real-Life Factors}

To provide a more practical and more accurate estimation on the amortized annual profit, we take the following factors into consideration.

\subsubsection{Maintenance Cost}

When one battery bank reaches its end-of-life, it should be replaced with a new one to keep the HEES system operational. The replacement process introduces the maintenance fee, since it requires maintenance personnel to come by and restore the system. We should also consider the maintenance fee in the installation of the HEES system.

\subsubsection{Discount Factor}

The discount factor reflects the time value of money, indicating that there is a difference between the future value of a payment and the present value of the same payment. The energy management system is a type of investment, of which the service life may be 10 years or longer, making the discount factor nonnegligible. Different from one time investments such as certificate of deposit (CD), the HEES system has replacement cost during operating. Therefore, amortizing the replacement cost should also consider the discount factor $\gamma$. This paper uses the annual percentage yield (2\%) of a 5-year $\mathrm{CD}$ as reference, and the discount factor is given by: $\gamma=1 /(1+2 \%)=0.9804$.

\subsubsection{System Form Factor}

Since the proposed energy management system is targeting residential usage, we must limit its overall form factor. In big cities such as the New York City where the housing price is extremely high, the residential HEES system should take no more volume than home electrical appliances (e.g., a refrigerator). In our problem formulation, we define the reciprocal of battery volumetric energy density as the unit volume, which is the volume of battery divided by the maximum stored energy. On average, the unit volume of lead-acid batteries is $12.5 \mathrm{~L} / \mathrm{kWh}$, which is much higher than that of Li-ion batteries: $2 \mathrm{~L} / \mathrm{kWh} 0$.

\subsection{Problem Formulation}

HEES system design requires careful determination of the capacities of both lead-acid and Li-ion battery banks, the SoC swing and DoD limits in both high season and low season, in order to maximize the amortized annual profit with the given budget and volume constraints. Taking all the aforesaid factors into consideration, we formulate this annual profit maximization problem as follows.

\section{Given:}

1) LUTs of high season energy cost reduction and low season cost reduction: $H T, L T$;

2) Unit price of Li-ion and lead-acid batteries: $p_{x}, p_{y}$;

3) Unit volume of Li-ion and lead-acid batteries: $v_{x}, v_{y}$;

4) One-time maintenance fee $M$ and discount factor $\gamma$;

5) Budget $B$ for initial investment and total volume limit $V$;

Find: Li-ion battery bank capacity $Q_{x}$ and maximum SoC swing $s_{x H}$ and $s_{x L}$ of high season and low season, respectively,, leadacid battery bank capacity $Q_{y}$ and maximum DoD $s_{y H}$ and $s_{y L}$ of high season and low season, respectively.

Maximize: amortized annual profit $A\left(Q_{x}, Q_{y}, s_{x H}, s_{y H}, s_{x L}, s_{y L}\right)$ Subject to:

1) Budget constraint: $Q_{x} p_{x}+Q_{y} p_{y}+M \leq B$;

2) System volume constraint: $Q_{x} v_{x}+Q_{y} v_{y} \leq V$.

The annual profit is the net profit the HEES system makes. In other words, we consider the HEES system as a long-term investment. The profit is the annual cost reduction of electricity bills subtracted by (i) the cost of the initial purchase of the HEES system, and (ii) the replacement cost (including the installation fee) of Li-ion battery banks and lead-acid battery banks. To determine the final return on investment, we assume that the HEES system operates for $L_{\text {sys }}$ years. Let $P_{i}$ denote the accumulative saving from the beginning to year $i$, accounting for the energy saving in both high season and low saving and capacity degradation. Whenever a battery bank reaches its end of life, it is replaced with a new one and the corresponding expenditure is subtracted from $P_{i}$. The discount factor is also considered to reflect the time value of money. Therefore, the relationship between $P_{i}$ and $P_{i-1}$ is given by

$P_{i}=P_{i-1} / \gamma+\widehat{F_{H}}\left(Q_{x i}, Q_{y i}, s_{x H}, s_{y H}\right)+\widehat{F_{L}}\left(Q_{x i}, Q_{y i}, s_{x L}, s_{y L}\right)$

$-R_{x i} \cdot\left(Q_{x} p_{x}+M\right)-R_{y i} \cdot\left(Q_{y} p_{y}+M\right)$

where $R_{x i}=1$ if the Li-ion bank is replaced in the $i$-th year and $R_{x i}=0$ otherwise; $R_{y i}=1$ if the lead-acid bank is replaced and $R_{y i}=0$ otherwise. The capacity values $Q_{x i}, Q_{y i}$ are updated each year according to the capacity degradation model. 
Let $L_{\text {sys }}$ denote the system lifetime and $A$ denote the amortized annual profit $A\left(Q_{x}, Q_{y}, s_{x H}, s_{y H}, s_{x L}, s_{y L}\right)$, we have:

$$
\begin{gathered}
A+A \cdot \gamma^{-1}+\cdots+A \cdot \gamma^{-L_{s y s}+1}=P_{L_{s y s}}, \\
A=P_{L_{s y s}} \cdot \frac{1-\gamma^{-1}}{1-\gamma^{-L_{s y s}}} .
\end{gathered}
$$

The value of $A$ converges as $L_{\text {sys }}$ approaches infinite.

In order to calculate $A$, we decide the time (year $i$ ) of battery bank replacement, i.e., $R_{x i}=1$ or $R_{y i}=1$. Unfortunately, the Liion lifetime model requires day-by-day recursive calculation to determine the current FCC, thereby making it too complicated to derive an analytical expression of its lifetime. Besides, the results of the DCR problem are stored in LUTs instead of given as an analytical expression. Therefore, it is impractical to derive an analytical solution to the global optimization problem. We use search-based algorithm to solve this annual profit maximization problem. However, since this calculation occurs only once in the entire HEES system lifetime (which can be over 20 years) to determine the system specifications, the high complexity of the search-based algorithm is acceptable.

\section{SIMULATION RESULTS}

Four energy storage systems are simulated and compared in this section:

1) An EES system with a Li-ion battery bank (or Li-ion EES);

2) An EES system with a lead-acid battery bank (or lead-acid EES);

3) A HEES system with the energy management policy in [11] (or no-buff HEES);

4) A HEES system with the proposed buffering-based energy management policy (or buffered HEES).

We show the results of daily optimization results, comparing the no-buff HEES and the buffered HEES under the time-of-day pricing policy. The annual profit results are shown next.

\subsection{Daily Optimization Results}

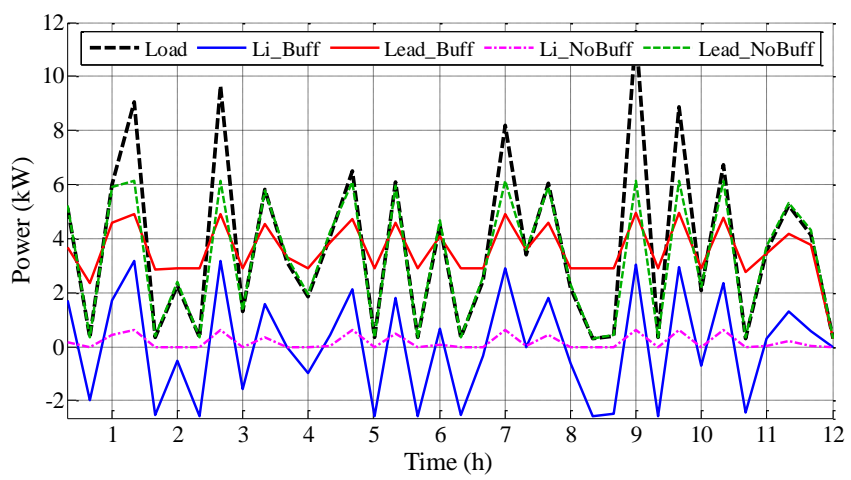

Figure 6. Comparison of the output power in peak hours.
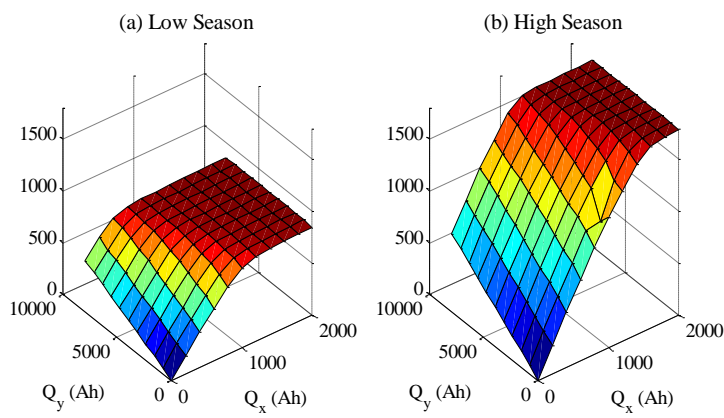

Figure 7. Maximum seasonal cost reduction results.
Figure 6 shows the output power as a function of time of the no-buff HEES and the buffered HEES, with the lead-acid battery bank capacity of $4 \mathrm{kAh}$ and the Li-ion battery bank capacity of 200Ah. The green curve and the pink curve depict the results of the no-buff system: Without buffering, the curve of lead-acid battery output power is largely influenced by the load profile. The instant output power can be restricted to almost zero at some decision epochs and ramp up to over $6.0 \mathrm{~kW}$ at some other epochs. The Li-ion battery bank gets discharged to cut the high load peaks but with very limited ability. On the other hand, when the Li-ion battery bank is allowed to get charged during peak hours (the blue curve), its ability to alleviate demand peaks gets improved, the output power peaks of the lead-acid battery bank are lowered (the red curve), and therefore the buffered HEES further reduces the energy loss due to rate capacity effect. As a results, the saving of the buffered HEES is improved by $6.10 \%$ compared to the no-buff HEES under time-of-day pricing for the load profile shown in Figure 6.

Figure 7 shows $F_{H S}\left(Q_{x}, Q_{y}\right)$ and $F_{L S}\left(Q_{x}, Q_{y}\right)$, the seasonal cost reduction results using time-of-day pricing and a multi-family unit electricity usage profile of 365 days in a year. As can be seen in Figure 7, the maximum seasonal energy cost reduction has diminishing marginal gain as the capacities increase.

\subsection{Global Optimization Results}

This subsection first provides the optimization results of the maximum annual profit with different budget and volume constraints under time-of-day pricing policy. Then, we compare the annual profits of the four aforesaid systems. The discussion over the change of annual profits caused by different pricing policies is provided at the last stage.

\subsubsection{Analysis of Optimization Results}

The optimization results of the proposed energy management system are provided in Table 3 in details. $A P$ is calculated by the ratio of annual profit to the initial budget. These results prove the conclusion that with a tight budget (e.g. $B=1000)$, larger lead-acid battery capacity should be adopted whereas a smaller space (e.g. 50L) allows a larger Li-ion battery bank. Considering the results of $s_{x H}, s_{y H}, s_{x L}$, and $s_{y L}$, we see that (i) full capacity of the leadacid battery bank should be used to maximize the cost saving in high season, and (ii) larger capacity of the Li-ion battery bank is involved in low season because lower and more demand valleys require more frequent energy buffering to maximize the daily saving.

Table 3. Detailed annual profit results.

\begin{tabular}{|c|c|c|c|c|c|}
\hline $\mathrm{B}(\$)$ & $\mathrm{V}(\mathrm{L})$ & $Q_{x}, Q_{y}(\mathrm{kWh})$ & $s_{x H}, s_{y H}, s_{x L}, s_{y L}$ & $A(\$)$ & $A P$ \\
\hline 1000 & 50 & $63.6,323$ & $0.5,1,1,0.5$ & 31.974 & $3.20 \%$ \\
\hline 3000 & 50 & 373,274 & $0.5,1,1,0.5$ & 42.877 & $1.43 \%$ \\
\hline 3000 & 100 & 285,621 & $0.5,1,1,0.5$ & 81.069 & $2.70 \%$ \\
\hline 5000 & 100 & 589,572 & $0.5,1,1,0.5$ & 90.357 & $1.81 \%$ \\
\hline
\end{tabular}

\subsubsection{Comparison Between Systems}

Figure 8 compares the derived maximum annual profits between Li-ion EES, lead-acid EES, no-buff HEES, and the buffered HEES under two different $(B, V)$ constraints and timeof-day pricing policy. The buffered HEES achieves the highest annual profit in both cases. For example, under $\$ 3000$ budget and $50 \mathrm{~L}$ volume constraint, the HEES unit achieves $192.4 \%$ more annual net profit than a Li-ion EES system and $42.65 \%$ more than a lead-acid EES system, $11.27 \%$ more than the no-buff HEES. 


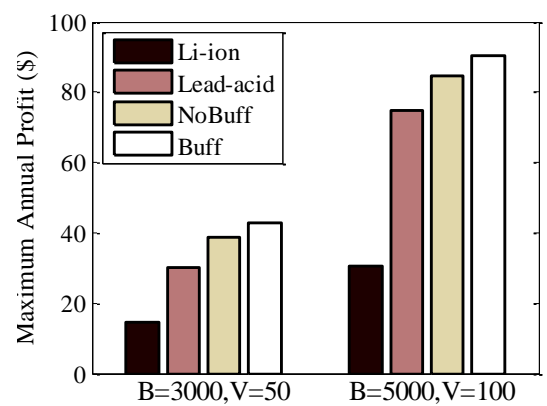

Figure 8. Maximum annual profit comparison of different systems under time-of-day pricing policy.

\subsubsection{Comparison Between Pricing Policies}

Apart from the currently existing pricing policy, we conduct experiments on different pricing policies to show the economic viability of the proposed system. This is important information for potential customers since (i) the electric energy pricing is always changing because of the increasing price of resource as well as inflation, and (ii) as is mentioned above, researchers in electrical energy area have been studying various pricing functions for the future smart grid. Therefore, we first explore the annual profit as a function of unit price difference between peak and off-peak hours and then explore the two pricing policies mentioned in Section 3.1.

We define $\rho \epsilon[0.7,1.1]$, and then multiply both the peak price and off-peak price of the New York City time-of-day pricing policy by $\rho$. The unit energy price difference between peak and off-peak is scaled by $\rho$ in this way. Figure 9 shows the annual profits under different budget and volumetric constraints. As is shown in this figure, the proposed system starts to make profits at between 0.8 to 0.9 of the original price difference, and grows dramatically as the difference increases.

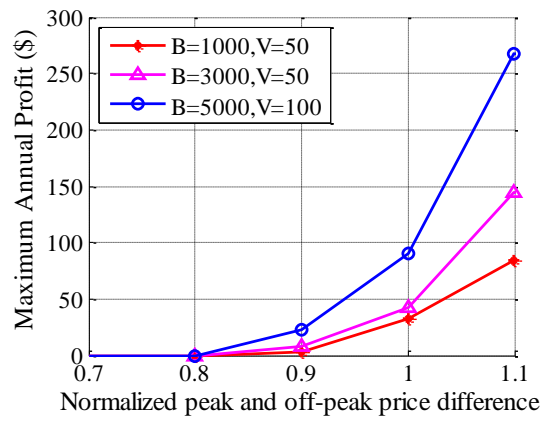

Figure 9. Maximum annual profit as a function of peak and off-peak price difference.

Table 4 provides the annual profits under two real-time pricing policies mentioned in Section 3.1. The profits is much higher compared to the results of time-of-day pricing policy in Table 3 , denoting that the proposed energy buffering system is able to bring more profits in real-time pricing policies, where the unit energy price increases with the increase of total energy consumption.

Table 4. Maximum annual profits of real-time pricing policies.

\begin{tabular}{|c|c|c|c|c|c|}
\hline \multirow{2}{*}{$\mathrm{B}(\$)$} & $\mathrm{V}(\mathrm{L})$ & \multicolumn{2}{|c|}{ Discrete } & \multicolumn{2}{c|}{ Continuous } \\
\cline { 3 - 6 } & & $A(\$)$ & $A P$ & $A(\$)$ & $A P$ \\
\hline 1000 & 50 & 438.50 & $43.85 \%$ & 312.16 & $31.22 \%$ \\
\hline 3000 & 50 & 706.27 & $23.54 \%$ & 549.69 & $18.32 \%$ \\
\hline 3000 & 100 & 1013.5 & $33.78 \%$ & 771.22 & $25.71 \%$ \\
\hline 5000 & 100 & 1226.1 & $24.52 \%$ & 971.96 & $19.44 \%$ \\
\hline
\end{tabular}

\section{CONCLUSIONS}

Lack of research on the profitability of hybrid electrical energy storage (HEES) systems makes residential users hesitate in deployment of HEES systems. In this paper, we present a comprehensive analysis on residential electric bill savings by employing an energy management system that consists of a HEES unit. We first derive an efficient energy management strategy for the HEES system on a daily basis and then give a near-optimal system design methodology to maximize amortized annual profits, under the budget and system volumetric constraints. We take both the New York City time-of-day pricing and real-time pricing policies with a one-year-long load profile of a multi-family dwelling unit and provide an in-depth case study. The results show that an optimally designed HEES system with energy buffering management obtains average $11.10 \%$ more benefits than the none-buffering HEES system under time-of-day pricing policy.

\section{REFERENCES}

[1] S. Borenstein: "The Trouble with Electricity Markets: Understanding California's Restructuring Disaster", The Journal of Economic Perspectives, 2002.

[2] Consolidated Edison Company of New York, Inc., "Service Classification No. 1 - Residential and Religious", 2012.

[3] H. Allcott, "Real time pricing and electricity markets." Harvard University, 2009.

[4] A.-H. Mohsenian-Rad and A. Leon-Garcia, "Optimal residential load control with price prediction in real-time electricity pricing environments", IEEE Transactions on Smart Grid, 2010.

[5] S. Yue, J. Chen, Y. Gu, C. Wu and Y. Shi, "Dual-pricing policy for controller-side strategies in demand side management", SmartGridComm, 2011.

[6] Y. Wang, S. Yue, L. Kerofsky, S. Deshpande, and M. Pedram, "A Hierarchical Control Algorithm for Managing Electrical Energy Storage Systems in Homes Equipped with PV Power Generation," Proc. of the IEEE Green Technologies Conference, Apr. 2012.

[7] M. Pedram, N. Chang, Y. Kim, Y. Wang, "Hybrid Electrical Energy Storage Systems", ISLPED, 2010.

[8] F. Koushanfar, "Hierarchical hybrid power supply networks", $D A C$, 2010.

[9] L. Exarchakos, M. Leach and G. Exarchakos, "Modelling electricity storage systems management under influence of demand-side management programmes", International Journal of Energy Research, 2009.

[10] C. Wei, H. Hu, Q. Chen and G. Yang, "Learning Agents for Storage Devices Management in the Smart Grid", CiSE, 2010.

[11] D. Zhu, Y. Wang, S. Yue, Q. Xie, N. Chang and M. Pedram, "Maximizing Return on Investment of a Grid-Connected Hybrid Electrical Energy Storage System", ASP-DAC, 2013.

[12] California $\quad$ ISO:
http://www.caiso.com/Pages/TodaysOutlook.aspx

[13] D. Linden, T. Reddy, Handbook of Batteries, McGraw-Hill Professional, 2001.

[14] MatLab R2012a Documentation: Optimization Toolbox http://www.mathworks.com/help/toolbox/optim/ug/fmincon.html

[15] A. Millner, "Modeling Lithium Ion Battery Degradation in Electric Vehicles," in CITRES, 2010.

[16] PNGV Test Plan for ATD 18650 Gen 1 Lithium-Ion Cells, Revision 4, EHV-TP-103, December 1999.

[17] M. Broussely, S. Herreyre, P. Biensan, P. Kasztejna, K. Nechev, R.J. Staniewicz, "Aging Mechanism in Li-ion Cells and Calendar Lfie Prediction", Journal of Power Sources, 2001.

[18] H. Bindner, T. Cronin, P. Lundsager, J.F. Manwell, U. Abdulwahid, I. Baring-Gould, "Lifetime Modeling of Lead Acid Batteries", Risø National Laboratory, 2005.

H. Chen, T. Cong, W. Yang, C. Tan, Y. Li, Y. Ding, "Progress in electrical energy storage system: A critical review", Progress in Natural Science, 2009. 